\title{
Perfect discrete Morse functions on 2-complexes
}

\author{
R. Ayala, D. Fernández-Ternero and J.A. Vilches \\ Dpto. de Geometría y Topología, Universidad de Sevilla, 41080, Sevilla, SPAIN \\ rdayala@us.es,desamfer@us.es,vilches@us.es
}

\begin{abstract}
This paper is focused on the study of perfect discrete Morse functions on a 2 -simplicial complex. These are those discrete Morse functions such that the number of critical $i$-simplices coincides with the $i$-th Betti number of the complex. In particular, we establish conditions under which a 2-complex admits a perfect discrete Morse function and conversely, we get topological properties of a 2-complex admitting such kind of functions. This approach is more general than the known results in the literature [7], since our study is not restricted to surfaces. These results can be considered as a first step in the study of perfect discrete Morse functions on 3-manifolds.
\end{abstract}

Keywords perfect discrete Morse function, Betti number, simplicial complex.

\section{Introduction}

Since it was introduced, Morse theory has been a powerful tool in the study of smooth manifolds by means of differential geometry techniques. Basically, it allows us to describe the topology of a manifold in terms of the cellular decomposition generated by the critical points of a scalar smooth map defined on it.

At the end of the last century, R. Forman [4] developed a discrete version of Morse theory that turned out to be a fruitful and efficient method for the study of the topology of discrete objects, such as simplicial and cellular complexes, which play a central role in many different fields of pure and applied mathematics.

Essentially, a discrete Morse function on a simplicial complex is a way to assign a real number to each simplex of a complex, without any continuity, in such a way that for each simplex the natural order given by the dimension simplices is respected, except at most in one (co)face of the given simplex. As in the smooth setting, changes in the topology of the level subcomplexes are deeply related to the presence of critical simplices of the function, and the analysis of the evolution of the homology of these complexes can be a very useful tool in computer vision to deal with shape recognition problems by means of topological shape descriptors. In our opinion, there are many advantages of using Forman's theory. First, it can be applied to discrete objects more general than manifolds. Second, it is more suitable in the digital context on topics like pattern recognition, shape classification and recognition, thinning 2D-objects where usually discretized functions are used.

Optimal discrete Morse functions has been widely studied in the literature [6, 7]. However, this question is not usually considered as an optimization problem in terms of obtaining discrete Morse functions with as less critical simplices as possible. On the contrary, this problem is mainly settled as a problem of perfect discrete Morse functions, that is, those functions satisfying $m_{i}(f)=b_{i}(M)$, where $m_{i}(f)$ is indicating the number of $i$-critical simplices of $f$ on $K$ and $b_{i}(K ; F)$ is the $i$-th Betti number of $M$. In this setting is interesting to point out that there are complexes which do not admit perfect discrete Morse functions. It can be explained by two main reasons: either a 
wrong selection of the field of coefficients of the Betti numbers (so it can be sorted out by choosing a suitable field) or the considered complex is homologically trivial (acyclic) but not homotopically trivial (non-contractible). In this sense, since we consider the problem on general 2-complexes, not just on surfaces as in [7], our results are useful to find examples of 2-complexes on which is not possible to define perfect discrete Morse functions. These kind of 2-complexes are interesting since the notions of perfect and optimal discrete Morse function are not equivalent on them.

\section{Preliminaries}

We recall that a CW-complex is built by gluing together certain basic building blocks called cells. A cell is a topological space that is homeomorphic to a ball.

The $n$-skeleton of a CW-complex is the union of the cells whose dimension is at most $n$.

A finite CW-complex is a space obtained by defining the $n$-skeleton inductively. We begin by taking the 0-skeleton to be a discrete space. Given the $(n-1)$-skeleton and a collection of closed $n$-cells, we define maps from the boundary $S^{n-1}$ of each $n$-cell into the $(n-1)$-skeleton. Next, we define the $n$-skeleton to be the identification space obtained from the union of the $(n-1)$-skeleton and the closed $n$-cells by identifying each point in the boundary of an $n$-cell with its image.

Let $K$ be a triangulated $n$-CW-complex and be a $n$-simplex of $K$. If there exists a $(n-1)$ dimensional face of such that is not a face of any other $n$-simplex in $K$, we say that there is an elementary collapse from $K$ to $K-\{$,$\} . The inverse operation is called an elementary$ expansion from $K-\{$,$\} to K$. If $K=K^{0} \quad K^{1} \quad \ldots \quad K^{m}=L$ are simplicial complexes such that there is an elementary collapse from $K^{i-1}$ to $K^{i}, i=1, \ldots, m$, we say that $K$ collapses to $L$, denoted by $K \quad L$. Equivalently, the inverse operation is called an expansion from $L$ to $K$, denoted by $K \quad L$.

From now on, if necessary, we will denote the dimension of a simplex by a super-index indicating it. Every Given a simplicial complex $K$, a discrete Morse function is a function $f: K-\mathbb{R}$ such that, for any $p$-simplex $K$ :

(M1) card $\{(p+1)>/ f() \leq f()\} \leq 1$.
$(\mathrm{M} 2) \operatorname{card}\{(p-1)</ f() \geq f()\} \leq 1$.

A $p$-simplex $\quad K$ is said to be a critical simplex with respect to $f$ if:

(C1) $\operatorname{card}\{(p+1)>/ f() \leq f()\}=0$.

(C2) $\operatorname{card}\{(p-1)</ f() \geq f()\}=0$.

A value of a discrete Morse function on a critical simplex is called critical value.

Given $c \mathbb{R}$, the level subcomplex $K(c)$ is the subcomplex of $K$ consisting of all simplices with $f() \leq c$, as well as all of their faces, that is,

$$
K(c)=\bigcup_{f(\tau) \leq c} \bigcup_{\sigma \leq \tau}
$$

Given two values of $f, a_{k}<a_{l}$, the relationship between two level subcomplexes $K\left(a_{k}\right)$ and $K\left(a_{l}\right)$ is the following (see $\left.[5]\right)$ :

- If the interval $\left[a_{k}, a_{l}\right]$ does not contain any critical value, then $K\left(a_{l}\right)$ collapses to $K\left(a_{k}\right)$ or equivalently, $K\left(a_{k}\right)$ expands to $K\left(a_{l}\right)$.

- If the interval $\left[a_{k}, a_{l}\right]$ contains a critical value corresponding to a critical simplex of dimension $i$, then $K\left(a_{l}\right)$ has the same simple homotopy type as $K\left(a_{k}\right)$ with an $i$-cell attached. 
A discrete vector field $V$ on $K$ is a collection of pairs $\left({ }^{(p)}<{ }^{(p+1)}\right)$ of simplices of $K$ such that each simplex is in at most one pair of $V$. A $V$-path is a sequence of simplices

$$
\stackrel{(p)}{0}, \quad \stackrel{(p+1)}{0}, \quad \stackrel{(p)}{1}, \quad{ }^{(p+1)}, \ldots, \quad \stackrel{(p+1)}{r}, \quad \underset{r+1}{(p)}, \ldots,
$$

such that, for each $i \geq 0$, the pair $\left({ }_{i}^{(p)}<{ }_{i}^{(p+1)}\right) \quad V$ and ${ }_{i}^{(p+1)}>{ }_{i+1}^{(p)}={ }_{i}^{(p)}$.

Given a discrete Morse function $f$ on $K$, the gradient vector field induced by $f$ is the set of pairs of simplices $\left({ }^{(p)}<(p+1)\right)$ such that $f() \geq f()$.

Theorem 2.1. [5] A discrete vector field $V$ is the gradient vector field of a discrete Morse function if and only if there are no non-trivial closed $V$-paths.

Theorem 2.2. [5] Let $f$ be a discrete Morse function defined on $K$ and let $b_{p}$ be the $p$-th Betti number of $K$ with $p=0,1, \ldots, n$ (where $n$ is the dimension of $K$ ). Then:

(I1) $m_{p}(f)-m_{p-1}(f)+\cdots \pm m_{0} \geq b_{p}-b_{p-1}+\cdots \pm b_{0}$,

(I2) $m_{p}(f) \geq b_{p}$

(I3) $m_{0}(f)-m_{1}(f)+\cdots+(-1)^{n} m_{n}(f)=b_{0}-b_{1}+\cdots+(-1)^{n} b_{n}=(K)$,

where $m_{p}(f)$ denotes the number of critical p-simplices of $f$ on $K$.

Notice that these inequalities are still valid for the case of Betti numbers with general coefficients, that is, using any field $F$ instead of $\mathbb{Z}$.

A discrete Morse function $f$ defined on $K$ is optimal if it has the least possible number of critical simplices, that is, $m_{i}(f) \leq m_{i}(g)$ with $1 \leq i \leq n$ for every discrete Morse function $g$ on $K$.

The Morse-Smale characteristic of a complex $K$ is defined as

$$
(K)=\min \left\{\mu(f): f \quad F_{M}(K)\right\}
$$

where $\mu(f)=\sum_{p=0}^{n} m_{p}(f)$ and $F_{m}(K)$ denotes the set of discrete Morse functions defined on $K$. Notice that $(K)=\mu(f)$ for all optimal discrete Morse function $f$.

In an analogous way we define the numbers ${ }_{i}(K)$ by

$$
{ }_{i}(K)=\min \left\{m_{i}(f): f \quad F_{M}(K)\right\}
$$

It is obvious that $(K) \geq \sum{ }_{i}(K)$ and $(K) \geq 1$.

Notice that if $K$ is a compact connected orientable surface without boundary then:

- $(K)$ is an even number.

- $(K)=2$ if and only if $K=S^{2}$.

A discrete Morse function $f$ is called $F$-perfect if $m_{p}(f)=b_{p}(K ; F)$ with $p=0, \ldots, n$ where $F$ is the field of coefficients used for computing the indicated Betti number.

Notice that every $F$-perfect discrete Morse function is optimal. However the converse is not true, that is, there exist optimal discrete Morse functions which are not $F$-perfect. Finally, we will introduce two examples of gradient vector fields on the torus and dunce hat respectively that illustrate the difference between both kind of functions. In particular, notice that the second example makes clear that the minimality of the number of critical simplices is not linked to the Betti numbers of the complex. 


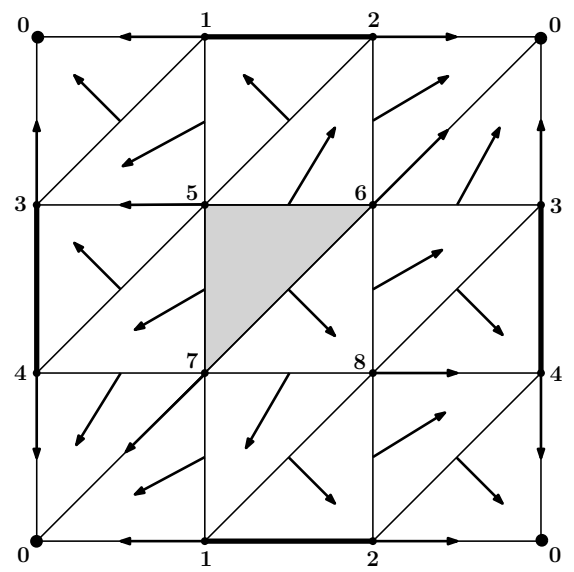

Figure 1: A gradient vector field on the torus induced by a perfect discrete Morse function.

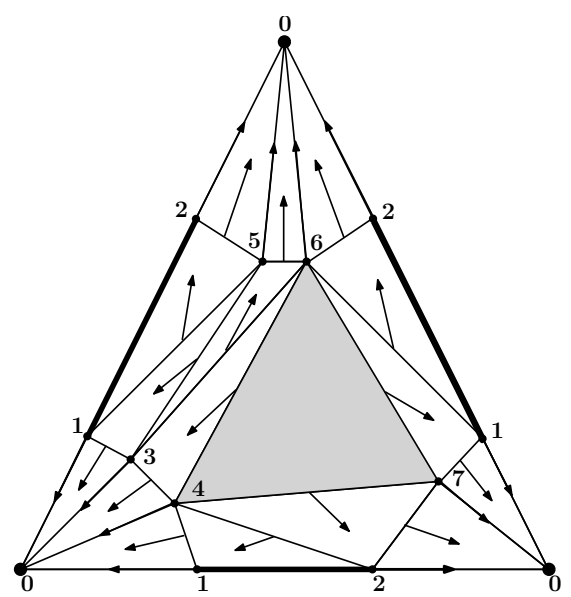

Figure 2: A gradient vector field on the dunce hat induced by an optimal (non-perfect) discrete Morse function.

\section{$3 \mathbb{Z}$-perfect discrete Morse functions on 2-complexes}

We will start the study of the existence of perfect discrete Morse functions on 2-complexes by considering the case of homology with integer coefficients.

Next result proves that every 1-dimensional complex admits $\mathbb{Z}$-perfect discrete Morse functions.

Proposition 3.1. Every connected graph admits a $\mathbb{Z}$-perfect discrete Morse function.

Proof. Let us consider a spanning tree $T$ in $G$. It is well known that $b_{1}(G)$ can be regarded as the number of edges $\left\{e_{1}, \ldots, e_{m}\right\}$ out of $T$. Let $v$ be any vertex of $T$. Since $T$ is collapsible to $V$ then, by means of Lemma 4.3 of [4], there is a discrete Morse function $g$ on $T$ such that $v$ is its unique critical simplex. Now, if we consider any edge $e_{i}$ not in $T$, by putting $f\left(e_{i}\right)>\max \left\{f\left(u_{i}\right), f\left(v_{i}\right)\right\}$ where $u_{i}$ and $v_{i}$ are the vertices of $e_{i}$, we get an extension $f$ of $g$ to $G$ with one critical vertex $v$ and $b_{1}(G)$ critical edges $e_{i}$.

Since the spanning tree is built in linear time and in the above proof every simplex is visited at most once, it is possible to define an algorithm which constructs a $\mathbb{Z}$-perfect discrete Morse function on $G$ whose time complexity is linear. 
The above proposition can be extended to any 2-complex which collapses to a graph in the following way

Corollary 3.2. Let $K$ be a 2-complex which collapses to a graph $G$ contained in $K$. Then $K$ admits a $\mathbb{Z}$-perfect discrete Morse function.

It is interesting to point out that since every surface with boundary collapses to a graph then such kind of surfaces admit a $\mathbb{Z}$-perfect discrete Morse function.

Next result shows us how collapsibility of a general complex can be put in terms of its MorseSmale characteristic.

Lemma 3.3. Let $K$ be complex. Then $K$ is collapsible if and only if $(K)=1$.

Proof. If $K$ is collapsible then the collapsing strategy gives us a discrete vector field which is induced by a discrete Morse function on $K$ with just a unique critical simplex (a critical vertex). Conversely, given a discrete Morse function on $K$ with just one critical simplex, its induced vector field can be interpreted as a collapsing strategy to a vertex in $K$.

Now we are going to give several results on the links between the existence of $\mathbb{Z}$-perfect discrete Morse functions on a given 2-complex with some trivial homology groups and its simple homotopy type.

Proposition 3.4. Let $K$ be a compact connected 2 -complex admitting a $\mathbb{Z}$-perfect discrete Morse function. The following results hold:

1. If $K$ is acyclic then it is collapsible.

2. If $H_{1}(K)=0$ and $H_{2}(K)=0$ then $K$ has the same simple homotopy type as a wedge of spheres $S^{2}$.

3. If $H_{1}(K)=0$ and $H_{2}(K)=0$ then $K$ has the same simple homotopy type as a graph.

Proof. 1. Since $K$ is acyclic and considering that it admits a $\mathbb{Z}$-perfect discrete Morse function $f$ then $m_{i}(f)=b_{i}(K ; \mathbb{Z})=0$ with $i=1,2$ and $m_{0}(f)=b_{0}(K ; \mathbb{Z})=1$. Thus, by Lemma 3.3 we conclude that $K$ is collapsible.

2. Since $H_{1}(K)=0, H_{2}(K)=0$ and taking into account that it admits a $\mathbb{Z}$-perfect discrete Morse function $f$ then $m_{i}(f)=0$ with $i=0,1$ and $m_{2}(f)=b_{2}(K ; \mathbb{Z}) \geq 1$. Thus, by means of Corollary 3.5 of [4], $K$ has the same simple homotopy as a wedge of $m_{2}(f) 2$-spheres.

3. Since $H_{1}(K)=0$ and $H_{2}(K)=0$ and considering that it admits a $\mathbb{Z}$-perfect discrete Morse function $f$ then $m_{i}(f)=0$ with $i=0,2$ and $m_{1}(f)=b_{1}(K ; \mathbb{Z}) \geq 1$. Thus, by means of Corollary 3.5 of $[4], K$ has the same simple homotopy as a graph.

Corollary 3.5. Let $K$ be a compact connected 2 -complex. If $K$ is acyclic and non-collapsible then $K$ does not admit $\mathbb{Z}$-perfect discrete Morse functions.

Let $c o(K)$ be the minimal number of 2 -simplices $1, \ldots, c o(K)$ that need to be removed from $K$ so that $K-\{1, \ldots, c o(K)\}$ collapses to a graph. Next theorem establishes how $\operatorname{co}(K)$ and the existence of $\mathbb{Z}$-perfect discrete Morse functions are related.

Theorem 3.6. Let $K$ be a compact connected 2 -complex. Then $K$ admits a $\mathbb{Z}$-perfect discrete Morse function if and only if $\operatorname{co}(K)=b_{2}(K ; \mathbb{Z})$. 
Proof. Let us suppose that $K$ admits a $\mathbb{Z}$-perfect discrete Morse function $f$. Thus $m_{i}(f)=b_{i}(K ; \mathbb{Z})$ with $i=0,1,2$, so, in particular, $m_{2}(f)=b_{2}(K ; \mathbb{Z})$. By removing the $m_{2}(f)$ critical 2-simplices we get a subcomplex $K^{\prime}$ with $b_{2}\left(K^{\prime} ; \mathbb{Z}\right)=0$. Notice that the restriction of $f$ to $K^{\prime}$ is a $\mathbb{Z}$-perfect discrete Morse function and hence, by Proposition 3.4, we obtain that $K^{\prime}$ has the same simple homotopy type as a graph, that is, $K^{\prime}$ collapses to a graph. Hence we get $\operatorname{co}(K) \leq b_{2}(K ; \mathbb{Z})$. In order to conclude that $\operatorname{co}(K)=b_{2}(K ; \mathbb{Z})$, let us assume that we remove $m 2$-simplices from $K$ with $m<m_{2}(f)$. In this case the resulting subcomplex $K^{\prime \prime}$ satisfies that $b_{2}\left(K^{\prime \prime} ; \mathbb{Z}\right) \geq 1$ and thus it does not collapse to a graph.

Conversely, let us suppose that $\operatorname{co}(K)=b_{2}(K ; \mathbb{Z})$. Let $G$ be the graph on which the subcomplex $K^{\prime}$ (obtained by removing $\operatorname{co}(K)$ 2-complexes from $K$ ) collapses. Starting from a $\mathbb{Z}$-perfect discrete Morse function $g$ on $G$, it can be extended to a $\mathbb{Z}$-perfect discrete Morse function $f^{\prime}$ on $K^{\prime}$. Finally, we extend $f^{\prime}$ to $K$ by assigning to every remaining 2 -simplex a value greater than all the values of its bounding 1-simplices and we obtain a $\mathbb{Z}$-perfect discrete Morse function on $K$.

Corollary 3.7. Let $K$ be a compact connected surface without boundary. Then $K$ admits a $\mathbb{Z}$-perfect discrete Morse function if and only if $K$ is orientable.

Proof. This results follows from the fact that every surface has $\operatorname{co}(K)=1$ (see [8]) and since $K$ is orientable then $b_{2}(K)=1$.

Remark 3.8. The above corollary can be extended in a straightforward way to 2-pseudomanifolds, that is, a 2-pseudomanifold $K$ admits a $\mathbb{Z}$-perfect discrete Morse function if and only if $K$ is orientable.

\section{$4 \quad F$-perfect discrete Morse functions on 2-complexes}

In this section we are going to introduce the general problem of the existence of $F$-perfect discrete Morse functions on 2-complexes where $F$ is any field. This problem arises in a natural way when we study why a given 2-complex does not admit $\mathbb{Z}$-perfect discrete Morse functions. It is essentially due to two main influences: either to the nature of the first fundamental group of the complex or to the existence of torsion elements in the first homology group of the complex.

In the first case we find examples as Dunce hat, Bing's house and more generally any acyclic and non-collapsible 2-complex. All of these complexes have in common that they are homologically but not homotopically trivial. In fact we are going to prove that these kind of complexes do not admit any kind of $F$-perfect Morse functions for any field $F$.

Theorem 4.1. If $K$ is an acyclic and non-collapsible connected 2 -complex then $K$ does not admit any $F$-perfect discrete Morse function for all field $F$.

Proof. Let us suppose that there exist a $F$-perfect discrete Morse function defined on $K$. Since $K$ is acyclic, we get that $b_{i}(K ; F)=0$ with $i=1,2$ and it implies that $f$ must satisfy $m_{i}(f)=0$ with $i=1,2$ and $m_{0}(f)=1$. Thus, by means of Lemma 3.3 we conclude that $K$ is collapsible, which is a contradiction.

Notice that in the second case, although these kind of complexes do not admit $\mathbb{Z}$-perfect discrete Morse functions, they admit $F$-perfect functions for a suitable field $F$. In particular, Corollary 3.7 characterizes those surfaces admitting $\mathbb{Z}$-perfect discrete Morse functions as orientable surfaces. However, next result shows the influence of a change of coefficients in the existence of perfect discrete Morse functions. 
Proposition 4.2. Any non-orientable compact connected surface without boundary admits a $\mathbb{Z}_{2}$ perfect function.

Proof. Let $K$ be a triangulated non-orientable compact connected surface without boundary. By removing a 2-simplex, we obtain a 2-complex $K^{\prime}$ which collapses to a graph $G$, so we can define a discrete Morse function $f^{\prime}$ on $K^{\prime}$ satisfying $m_{0}\left(f^{\prime}\right)=1, m_{1}\left(f^{\prime}\right)=h$ and $m_{2}\left(f^{\prime}\right)=0$. Repeating the argument of the converse of the proof of Theorem 3.6, we obtain a discrete Morse function $f$ on $K$ with $m_{0}(f)=1, m_{1}(f)=h$ and $m_{2}(f)=1$. Finally, since $b_{0}\left(K ; \mathbb{Z}_{2}\right)=1, b_{1}\left(K ; \mathbb{Z}_{2}\right)=h$ and $b_{2}\left(K ; \mathbb{Z}_{2}\right)=1$, we conclude that $f$ is a $\mathbb{Z}_{2}$-perfect discrete Morse function.

Corollary 4.3. Any compact connected surface admits a $\mathbb{Z}_{2}$-perfect function.

Remark 4.4. Moreover, any pseudo-projective space, that is, the space obtained by gluing a 2-ball to $S^{1}$ by means of a map of degree $p$, admits $\mathbb{Z}_{p}$-perfect functions. It can be proved by repeating the argument of the proof of Proposition 4.2.

\section{Conclusions and future work}

The existing results in the literature on optimal discrete Morse functions are mainly restricted to triangulated surfaces. In this sense, our work extends this study to the case of general simplicial 2-complexes. In our opinion, this point of view is interesting for two main reasons: First, in our setting the notions of optimal and perfect discrete Morse functions are not equivalent. Then a new problem arises: how to determine an optimal discrete Morse function on a given 2-complex, for example, on an acyclic non-contractible one. On the other hand, our approach can be regarded as a first step in the study of perfect discrete Morse functions on triangulated 3-manifolds. It can be carried out by collapsing the considered 3-manifold to a 2-complex, the so called spine of the 3 -manifold, and then we reduce the problem to get a perfect function on a 2-complex.

\section{References}

[1] D. Andrica. Critical point theory and some applications. Cluj University Press, Romania, 2005.

[2] R. Ayala, L. M. Fernández, and J. A. Vilches. Characterizing equivalent discrete Morse functions. Bull. Braz. Math. Soc. (N.S.), 40(2):225-235, 2009.

[3] R. Ayala, L. M. Fernández, and J. A. Vilches. The number of critical elements of discrete Morse functions on non-compact surfaces. Topology Appl., 157(1):90-101, 2010.

[4] R. Forman. Morse Theory for cell complexes. Adv. Math., 134(1):90-145, 1998.

[5] R. Forman. A user's guide to discrete Morse theory. Sém. Lothar. Combin., 48, 2002. Art. B48c, 35 pp.

[6] P. Hersh. On optimizing discrete Morse functions. Adv. in Appl. Math., 35(3):294-322, 2005.

[7] T. Lewiner, H. Lopes, and G. Tavares. Optimal discrete Morse functions for 2-manifolds. Comput. Geom., 26(3):221-233, 2003.

[8] J. R. Munkres. Elements of Algebraic Topology. Addison-Wesley, Menlo Park, 1984. 
